\title{
Low local blood perfusion, high white blood cell and high platelet count are associated with primary tumor growth and lung metastasis in a 4T1 mouse breast cancer metastasis model
}

\author{
CHUAN WANG $^{1 *}$, YING-GE CHEN ${ }^{1 *}$, JIAN-LI GAO ${ }^{1}$, GUI-YUAN LYU ${ }^{1}$, JIE SU $^{1}$, QI ZHANG ${ }^{2}$, XIN JI $^{3}$, \\ JI-ZHONG YAN $^{4}$, QIAO-LI QIU ${ }^{1}$, YUE-LI ZHANG ${ }^{2}$, LIN-ZI LI $^{2}$, HAN-TING XU ${ }^{1}$ and SU-HONG CHEN ${ }^{2}$ \\ ${ }^{1}$ Institute of Materia Medica, Zhejiang Chinese Medical University, Hangzhou, Zhejiang 310053; \\ ${ }^{2}$ Institute of Chinese Medicine, Wenzhou Medical University, Wenzhou, Zhejiang 325035; \\ ${ }^{3}$ Department of Pharmacology, School of Medicine, Zhejiang University, Hangzhou, Zhejiang 310058; \\ ${ }^{4}$ College of Pharmaceutical Science, Zhejiang University of Technology, Hangzhou, Zhejiang 310014, P.R. China
}

Received August 4, 2014; Accepted April 20, 2015

DOI: $10.3892 / 01.2015 .3304$

\begin{abstract}
It was originally thought that no single routine blood test result would be able to indicate whether or not a patient had cancer; however, several novel studies have indicated that the median survival and prognosis of cancer patients were markedly associated with the systemic circulation features of cancer patients. In addition, certain parameters, such as white blood cell (WBC) count, were largely altered in malignant tumors. In the present study, routine blood tests were performed in order to observe the change of blood cells in tumor-bearing mice following the implantation of 4T1 breast cancer cells into the mammary fat pad; in addition, blood flow in breast tumor sites was measured indirectly using laser Doppler perfusion imaging (LDPI), in an attempt to explain the relevance between the blood circulation features and the growth or metastasis of
\end{abstract}

Correspondence to: Dr Jian-Li Gao, Institute of Materia Medica, Zhejiang Chinese Medical University, 548 Binwen Road, Binjiang, Hangzhou, Zhejiang 310053, P.R. China

E-mail: jianligao@gmail.com

*Contibuted equally

Abbreviations: Dox, doxorubicin; WBC, white blood cells; RBC, red blood cells; HGB, hemoglobin; HCT, hematocrit; MCV, mean corpuscular volume; $\mathrm{MCH}$, mean corpuscular hemoglobin; $\mathrm{MCHC}$, mean corpuscular hemoglobin concentration; RDW-SD, red blood cell distribution width by standard deviation; RDW-CV, red blood cell distribution width by coefficient of variation; MPV, mean platelet volume; PLT, platelets; PCT, plateletcrit; EO\%, ratio of eosinophils; $\mathrm{BASO} \%$, ratio of basophils; EO\#, number of eosinophils; BASO\#, number of basophils; PDW, platelet distribution width; P-LCR, platelet-large cell ratio; PDGF, platelet-derived growth factor; LDPI, laser Doppler perfusion imaging

Key words: circulate blood perfusion, basophils, thrombosis, breast cancer, lung metastasis breast cancer in mice model. The LDPI and blood test results indicated that the implantation of 4T1 breast cancer cells into $\mathrm{BALB} / \mathrm{c}$ mice led to thrombosis as well as high WBC count, high platelet count, high plateletcrit and low blood perfusion. Following implantation of the 4T1 cells for four weeks, the lung metastatic number was determined and the Pearson correlation coefficient revealed that the number of visceral lung metastatic sites had a marked negative association with the ratio of basophils (BASO\%; $\mathrm{r}=-0.512 ; \mathrm{P}<0.01$ ) and the mean corpuscular hemoglobin was significantly correlated with primary tumor weight $(\mathrm{r}=0.425 ; \mathrm{P}<0.05)$. In conclusion, the results of the present study demonstrated that tumor growth led to thrombosis and acute anemia in mice; in addition, when blood BASO\% was low, an increased number of lung metastases were observed in tumor-bearing mice.

\section{Introduction}

Over the past several decades, developments in clinical and surgical treatments for cancer patients has led to increased overall survival rates (1). However, the growth of primary tumors and subsequent cancer metastases continue to cause mortality and prevent effective treatment (2). Vascular dissemination is a major mechanism by which breast cancer cells migrate into the systemic circulation, leading to distant metastasis and mortality (3). Clinical observations have indicated a potential association between the bloodstream and cancer metastasis (4-5). However, few studies have investigated the systemic circulation characterization in mouse breast cancer metastasis models, which therefore limits the development of experimental oncology research.

Cancer patients have frequently been reported to present with symptoms of thrombosis, which are more severe if the disease has progressed to a metastatic stage $(6,7)$. In order for metastases to form, cancer cells must be able to survive in the harsh circulatory environment and extravasate into distant sites. Thrombosis may improve the anchoring of 'seed' (tumor cells that have escaped surgical removal or that may have already disseminated) to 'soil' (target organ) (8). Therefore, 
the elucidation of tissue perfusion of cancer organs or tissues is essential for predicting cancer growth and metastasis in tumor-bearing animals or in human cancer patients; in addition, there may be great clinical significance in planning treatment schedules in patients with vein tumor thrombosis or microcirculatory disorders. Laser Doppler perfusion imaging (LDPI) is a non-contact real-time laser imaging technique, which is used to measure tissue perfusion and is characterized as a non-invasive and non-ionizing technique. LDPI provides a highly sensitive, simple and inexpensive tool for blood perfusion detection of surface cancer tissue (9). The present study was performed in order to investigate the thrombosis of tumor-bearing mice using LDPI in combination with blood cell analysis.

Platelets are essential mediators of the process of pathologic thrombosis (10). Previous experimental evidence has demonstrated that platelets support tumor metastasis (11). Platelet activation and the coagulation system have been demonstrated to have a critical role in cancer progression (12). The role of platelets in the circulatory system is to protect tumor cells from immune elimination and promote their arrest at the endothelium, which promotes the formation of secondary lesions. This demonstrates that platelets contribute to tumor cell survival and metastasis, which therefore indicates that platelets may provide a novel therapeutic avenue for anti-metastatic cancer treatment (13).

High white blood cell (WBC) count has been reported to be more frequent in cancer patients with metastasis (14). WBCs are produced by bone marrow and a high WBC count is indicative of inflammation, trauma, allergies, leukemia or infections; however, this may also occur due to intense exercise (15). There are five types of WBCs, including neutrophils, lymphocytes, monocytes, eosinophils and basophils, which each serve a different function in the body. Therefore, the elevated count of a specific type of WBC may be suggestive of a specific disease. For instance, high monocyte levels are indicative of bacterial infections (16), while high neutrophil levels are associated with poor prognosis in malignant mesothelioma (17).

A previous study indicated a significant change in the systemic circulation characterization of a 4T1 mouse breast cancer metastasis model (9). Therefore, the present study aimed to evaluate the correlation among the number of visceral lung metastatic sites, primary tumor weight and the distribution of circulating blood cells in vivo in order to demonstrate the influence of cancer growth and metastasis on blood cell composition and perfusion in a mouse breast cancer model.

\section{Materials and methods}

Cell culture and animals. 4T1-Luc mouse breast cancer cells were provided by Professor Tong-Chuan He (University of Chicago Medical Center, Chicago, IL, USA) (9) and the wild-type 4T1 cell line was purchased from the American Type Culture Collection (Manassas, VA, USA), the cells were grown in Dulbecco's modified Eagle's medium (Invitrogen Life Technologies, Carlsbad, CA, USA) supplemented with $10 \%$ fetal bovine serum (Hyclone Laboratories, Inc., Logan, UT, USA) and 50 Units penicillin/streptomycin (Genom
Bio-pharmaceutical Tech, Hangzhou, China). All cells were cultured at $37^{\circ} \mathrm{C}$ in a $5 \% \mathrm{CO}_{2}$ incubator. $0.25 \%$ trypsin and $0.02 \%$ EDTA were purchased from Genom Bio-pharmaceutical Tech. Co., Ltd. (Hangzhou, China).

Female BALB/c mice (4-5 weeks old) were purchased from Shanghai Laboratory Animal Research Center (Shanghai, China) and maintained at the animal facility of the Experimental Animal Research Center of Zhejiang Chinese Medical University (Hangzhou, China). All procedures were performed according to the Guidelines for the Use and Care of Laboratory Animals published by the Zhejiang province (2009) (18). The study was approved by the ethics committee of Zhejiang Chinese Medical University (Hangzhou, China).

$4 T 1$ tumor-bearing mice model. Female BALB/c mice (4 weeks old, $\mathrm{n}=30$; weight, $18-20 \mathrm{~g} ; \mathrm{n}=5 \mathrm{mice} / \mathrm{group}$ ) were maintained in a pathogen-free environment with access to food and water ad libitum. Subconfluent 4T1-Luc cells were harvested, resuspended in phosphate-buffered saline (PBS) (Sigma-Aldrich, St .Louis, MO, USA) and analyzed using a $0.4 \%$ trypan blue exclusion assay (Beijing Solarbio Science \& Technology Co., Ltd., Shanghai, China; viable cells, $>95 \%$ ). For the breast cancer cell injection, $\sim 5 \times 10^{5} 4 \mathrm{~T} 1-\mathrm{Luc}$ cells in $100 \mu \mathrm{l}$ PBS were injected into the mammary fat pad (MFP) of each mouse using 27-gauge needles (Zhejiang Yusheng Medical Instrument Co.,Ltd., Jiaxing, China) (9). Doxorubicin hydrochloride (Dox; $10 \mathrm{mg}$ ) was purchased from Pfizer (New York, NY, USA) and dissolved in $1 \mathrm{ml}$ sterilized saline (Puaisi Medical Co., Ltd., Taizhou, China), then stored at $-20^{\circ} \mathrm{C}$. At $48 \mathrm{~h}$ following tumor cell injection, Dox ( $1 \mathrm{mg} / \mathrm{kg} / 2$ days) was orally administered to mice in the Dox group. The dimensions of the primary tumor sites were measured every 3-4 days using vernier calipers. Tumor volume was calculated using the following equation: Volume $=$ [length (L) + width (W) $]$ x L x W x 0.2618 (19). At the endpoint (4 weeks post treatment), mice were sacrificed by carbon dioxide asphyxiation, followed by cervical dislocation. Primary tumors were retrieved and weighed, and lung metastases were counted.

Blood samples and routine blood tests. For laboratory measurements, $100 \mu \mathrm{l}$ mouse whole blood was collected into tubes containing EDTA (Genom Bio-pharmaceutical Tech, Hangzhou, China), an in vitro anticoagulant, prior to sacrifice. Routine blood tests were immediately performed using a Sysmex XT-2000i automated hematology analyzer (Sysmex Corp., Hyogo, Japan) for the following parameters: WBCs, red blood cells (RBCs), hemoglobin (HGB), hematocrit (HCT), mean corpuscular volume (MCV), mean corpuscular hemoglobin $(\mathrm{MCH})$, mean corpuscular hemoglobin concentration (MCHC), red blood cell distribution width by standard deviation (RDW-SD), red blood cell distribution width by coefficient of variation (RDW-CV), mean platelet volume (MPV), platelets (PLT), plateletcrit (PCT), ratio of eosinophils [(number of eosinophils / number of white blood cell $) \times 100 \%,(\mathrm{EO} \%)]$, ratio of basophils [(number of basophils / number of white blood cell) $\times 100 \%$ (BASO\%)], number of eosinophils (EO\#), number of basophils (BASO\#), platelet distribution width (PDW) and platelet-large cell ratio (P-LCR). 

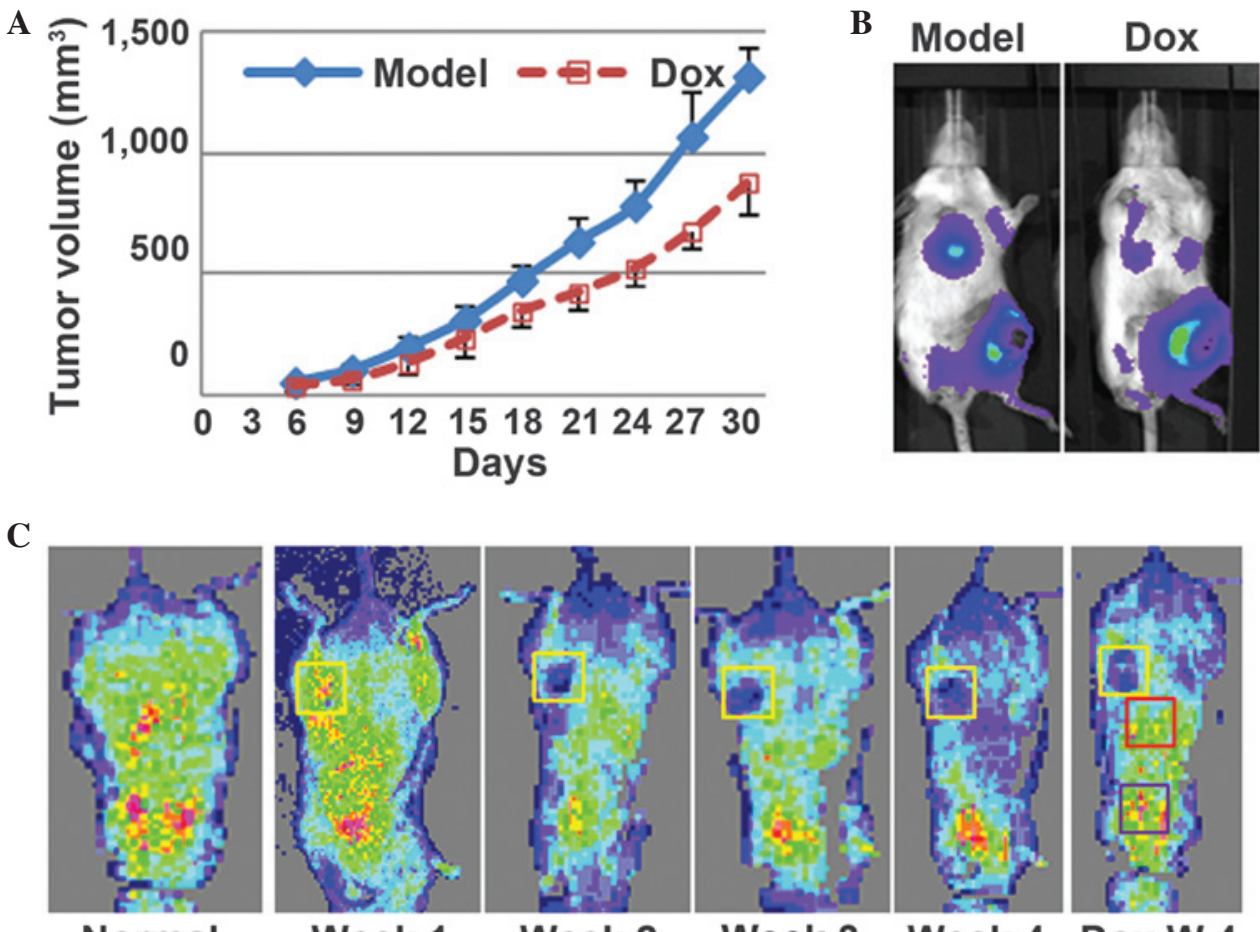

Normal
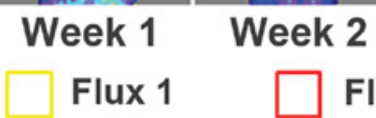

Week 3

Week 4

Dox W 4

Flux 3

Figure 1. Tumor volume, metastasis and blood perfusion of 4T1-tumor-bearing mice. (A) Tumor volume of model mice and Dox-treated tumor-bearing mice. 4T1-Luc cells were collected and injected into the mammary fat pad of BALB/c mice $\left(5 \times 10^{5}\right.$ cells/injection; $\mathrm{n}=5$ mice/group). Animals in the Dox treatment group were intraperitoneally injected with $1 \mathrm{mg} / \mathrm{kg} / 2$ days Dox for 4 weeks and then sacrificed, the model group was untreated and sacrificed at 4 weeks posttumor cell implantation. Tumor volume was detected every 3-4 days. Values are presented as the mean \pm standard deviation. (B) Bioluminescence imaging of model (left) and Dox-treated (right) tumor-bearing mice following 28 days of treatment. Representative Xenogen imaging results are shown. (C) In vivo laser Doppler perfusion imaging of mice. Representative images of the normal group, model group in week 1, week 2, week 3, week 4 and Dox-treated group at week 4 are shown. Dox, doxorubicin hydrochloride; Flux 1, blood flow at tumor site; Flux 2, blood flow in adjacent healthy skin; Flux 3, blood flow in mice hearts.

Xenogen bioluminescence imaging. Whole-body optical imaging of the mice was performed as previously described (20). Briefly, mice were anesthetized with isoflurane $(2 \%, 1.5 \mathrm{l} / \mathrm{min})$ via a nose-cone mask within a Xenogen IVIS 200 imaging system (Caliper Life Sciences, Hopkinton, MA, USA). This in vivo imaging was performed weekly following MFP injection for 4 weeks prior to sacrifice in treated and untreated mice.

For imaging, mice were administered an intraperitoneal injection of $100 \mathrm{mg} / \mathrm{kg}$ body weight D-luciferin sodium salt (Gold Biotechnology, Inc., St. Louis, MO, USA) in $0.1 \mathrm{ml}$ sterile PBS. Images were acquired through superimposing the emitted light over the grayscale photographs of the mice. Xenogen's Living Image V2.50.1 software (Caliper Life Sciences) was used for quantitative analysis, as described previously (21). Tumor, lung and vascular samples were retrieved for histological examination.

High-resolution LDPI. Micro-vascular blood flow was evaluated using a laser Doppler FLPI-CH with moorFLPI V2.1 software (Moor Instruments, Axminster, UK) (9). Hair was carefully removed from certain areas of the mice to allow for efficient scanning; mice were then anesthetized with isoflurane $(2 \%, 1.51 / \mathrm{min})$ via a nose-cone mask. The distance between the scanner and the skin surface was $10 \mathrm{~cm}$. Three examined areas $\left(1.4 \mathrm{~cm}^{2}\right)$ were selected in order to evaluate blood flow in the tumor (Flux 1), adjacent healthy skin (Flux 2) and heart of the mice (Flux 3). Erythrocytes reflect the laser beam, which enables the recording of the returning signal using a detector positioned in the scanner head. The returning signal is converted to an electrical signal, which is proportional to tissue perfusion (22). The underlying perfusion intensity values are expressed according to the diVerent color scale (23), extending from blue (low perfusion values) over green and yellow to red (highest perfusion values). The associated perfusion value was calculated as follows: Perfusion rate $=[($ Flux 1 or Flux 2)/Flux 3] x 100\%.

Statistical analysis. All data are expressed as mean \pm standard deviation and were subjected to one-way analysis of variance. The correlation coefficient was analyzed using Pearson's correlation coefficient. All statistical analyses were performed using SPSS 17.0 software (SPSS, Inc., Chicago, IL, USA). $\mathrm{P}<0.05$ was considered to indicate a statistically significant difference between values.

\section{Results}

Primary tumor volume, weight and lung metastatic sites. The present study first evaluated the primary tumor volume, weight and lung metastatic number of the 4T1 mouse breast cancer tumor-bearing mice. Tumor growth was monitored through measuring the tumor volume (Fig. 1A) and bioluminescence was detected using whole body Xenogen imaging, which 
Table I. Tumor weight and lung metastasis of 4T1 tumor-bearing and normal mice.

\begin{tabular}{lccccc}
\hline Group/Parameter & Tumor weight $(\mathrm{g})$ & Tumor index & Lung metastasis sites & Flux 1 (week 3) & Flux 2 (week 3) \\
\hline Normal & - & - & - & $0.97 \pm 0.12$ & $0.98 \pm 0.09$ \\
Model & $0.85 \pm 0.13$ & $0.04 \pm 0.01$ & $6.20 \pm 3.71$ & $0.50 \pm 0.09^{\mathrm{b}}$ & $0.79 \pm 0.15^{\mathrm{a}}$ \\
Dox & $0.71 \pm 0.05^{\mathrm{c}}$ & $0.04 \pm 0.00$ & $6.80 \pm 5.27$ & $0.61 \pm 0.09$ & $0.73 \pm 0.06$ \\
\hline
\end{tabular}

Values are presented as the mean \pm standard deviation ( $\mathrm{n}=5$ mice/group). ${ }^{\mathrm{a}} \mathrm{P}<0.05$ and ${ }^{\mathrm{b}} \mathrm{P}<0.01$ vs. normal group, ${ }^{\mathrm{c}} \mathrm{P}<0.05$ vs. model group. Flux 1, blood flow at tumor site or identical site on normal group; Flux 2, blood flow in adjacent normal tissue; Dox, doxorubicin hydrochloride.

Table II. WBC parameters in 4T1 breast cancer and normal mice.

\begin{tabular}{lccccc}
\hline Group/Parameter & WBC $\left(10^{9} / 1\right)$ & EO $(\%)$ & EO\# $\left(10^{9} / 1\right)$ & BASO $(\%)$ & BASO\# $\left(10^{9} / 1\right)$ \\
\hline Normal & $10.24 \pm 1.57$ & $1.02 \pm 0.32$ & $0.11 \pm 0.04$ & $0.04 \pm 0.05$ & $0.0004 \pm 0.0005$ \\
Model & $313.49 \pm 114.69^{\mathrm{b}}$ & $0.24 \pm 0.20^{\mathrm{b}}$ & $0.57 \pm 0.45$ & $0.34 \pm 0.14^{\mathrm{b}}$ & $0.1240 \pm 0.0872^{\mathrm{a}}$ \\
Dox & $197.61 \pm 37.03$ & $0.30 \pm 0.26$ & $0.50 \pm 0.37$ & $0.22 \pm 0.07$ & $0.0422 \pm 0.0153$
\end{tabular}

Values are presented as the mean \pm standard deviation ( $\mathrm{n}=5$ mice/group). ${ }^{\mathrm{a}} \mathrm{P}<0.05$ and ${ }^{\mathrm{b}} \mathrm{P}<0.01$ vs. normal group. WBC, white blood cell; $\mathrm{EO}$, eosinophils; BASO, basophils; Dox, doxorubicin hydrochloride.

Table III. RBC parameters in 4T1 breast cancer and normal mice.

\begin{tabular}{lclll} 
A, & & & & \\
\hline Group/Parameter & RBC $\left(10^{12} / 1\right)$ & HGB $(\mathrm{g} / \mathrm{l})$ & HCT $(\%)$ & MCH $(\mathrm{pg})$ \\
\hline Normal & $11.11 \pm 0.44$ & $166.60 \pm 3.38$ & $48.16 \pm 3.33$ & $15.02 \pm 0.61$ \\
Model & $9.51 \pm 0.73^{\mathrm{b}}$ & $135.80 \pm 10.42^{\mathrm{b}}$ & $42.62 \pm 3.07^{\mathrm{a}}$ & $14.30 \pm 0.14^{\mathrm{a}}$ \\
Dox & $9.42 \pm 0.28$ & $143.20 \pm 3.54$ & $43.72 \pm 0.96$ & $15.20 \pm 0.13^{\mathrm{c}}$ \\
\hline
\end{tabular}

B,

\begin{tabular}{|c|c|c|c|c|}
\hline Group/Parameter & $\mathrm{MCHC}(\mathrm{g} / \mathrm{l})$ & RDW-SD (fL) & RDW-CV (\%) & MCV (fL) \\
\hline Normal & $347.80 \pm 28.22$ & $29.22 \pm 1.71$ & $22.52 \pm 0.48$ & $43.30 \pm 1.74$ \\
\hline Model & $318.60 \pm 3.72$ & $29.84 \pm 0.38$ & $21.60 \pm 0.59^{\mathrm{a}}$ & $44.82 \pm 0.44$ \\
\hline Dox & $327.60 \pm 2.24^{\mathrm{c}}$ & $37.40 \pm 2.10^{c}$ & $24.62 \pm 1.03^{c}$ & $46.40 \pm 0.49^{c}$ \\
\hline
\end{tabular}

Values are presented as the mean \pm standard deviation ( $\mathrm{n}=5$ mice/group). ${ }^{\mathrm{a}} \mathrm{P}<0.05$ and ${ }^{\mathrm{b}} \mathrm{P}<0.01$ vs. normal group, ${ }^{\mathrm{c}} \mathrm{P}<0.05$ vs. model group. RBC, red blood cell; HGB, hemoglobin; HCT; hematocrit; MCH, mean corpuscular hemoglobin; MCHC, MCH concentration; RDW-SD, red blood cell distribution width by standard deviation; RDW-CV, red blood cell distribution width by coefficient of variation; MCV, mean corpuscular volume; Dox, doxorubicin hydrochloride.

demonstrated that the model and Dox-treated groups exhibited a marked Xenogen imaging signal in the lungs. The results revealed that Dox significantly inhibited primary tumor growth at 4 weeks following treatment (Fig. 1A); however, the number of metastasis sites on the lung surface of the Dox-treated group was not decreased compared with the control group at 4 weeks following treatment.

Characterization of blood perfusion in tumor-bearing mice. The overall perfusion of mice was decreased following tumor cell implantation (Fig. 1C), particularly at week 2 . The perfusion values for the primary tumor, Flux 1, were found to be associated with tumor size. When the diameter of the tumors was $<5 \mathrm{~mm}$ (week 1), Flux 1 was comparable to that of the average perfusion of normal mice at the same site (Fig. 1C). A lower perfusion intensity at the center of tumors was observed in the cases of tumors with a diameter $>7 \mathrm{~mm}$ (mean perfusion values: Model group, 0.51 \pm 0.11 ; and normal group, $0.65 \pm 0.05$ ) (Fig. 1C, week 2). This may be due to the development of a central necrosis. The perfusion value of the peripheral zone of primary 
Table IV. Platelet parameters in 4T1 breast cancer and healthy mice.

\begin{tabular}{lccccc}
\hline Group/Parameter & PLT $\left(10^{9} / 1\right)$ & PCT $(\%)$ & PDW (fL) & MPV (fL) & P-LCR (\%) \\
\hline Normal & $1017.60 \pm 139.03$ & $0.66 \pm 0.09$ & $7.52 \pm 0.27$ & $6.52 \pm 0.16$ & $4.98 \pm 0.89$ \\
Model & $1274.20 \pm 52.14^{\mathrm{b}}$ & $0.91 \pm 0.04^{\mathrm{b}}$ & $8.26 \pm 0.29^{\mathrm{b}}$ & $7.14 \pm 0.14^{\mathrm{b}}$ & $6.88 \pm 0.98^{\mathrm{a}}$ \\
Dox & $954.20 \pm 318.02$ & $0.68 \pm 0.22$ & $8.48 \pm 0.17$ & $7.16 \pm 0.12$ & $7.68 \pm 1.04$
\end{tabular}

Values are presented as the mean \pm standard deviation ( $\mathrm{n}=5$ mice/group). ${ }^{\mathrm{a}} \mathrm{P}<0.05$ and ${ }^{\mathrm{b}} \mathrm{P}<0.01 \mathrm{vs}$. normal group. PLT, platelet; PCT, plateletcrit; PDW, platelet distribution width; MPV, mean platelet volume; P-LCR\%, platelet-large cell ratio; Dox, doxorubicin hydrochloride.

A
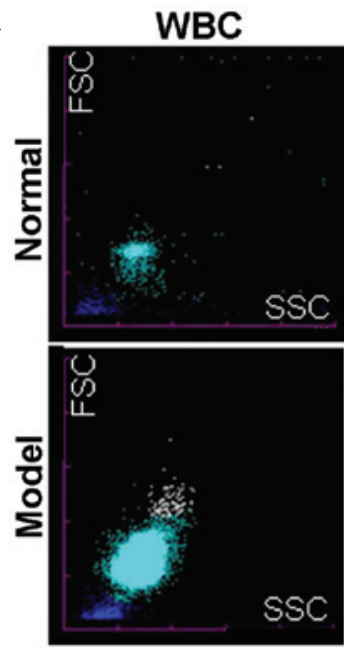

B

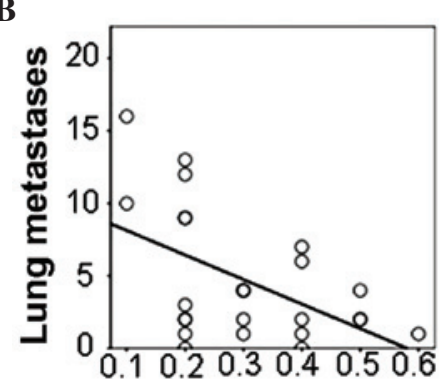

BASO $\%$
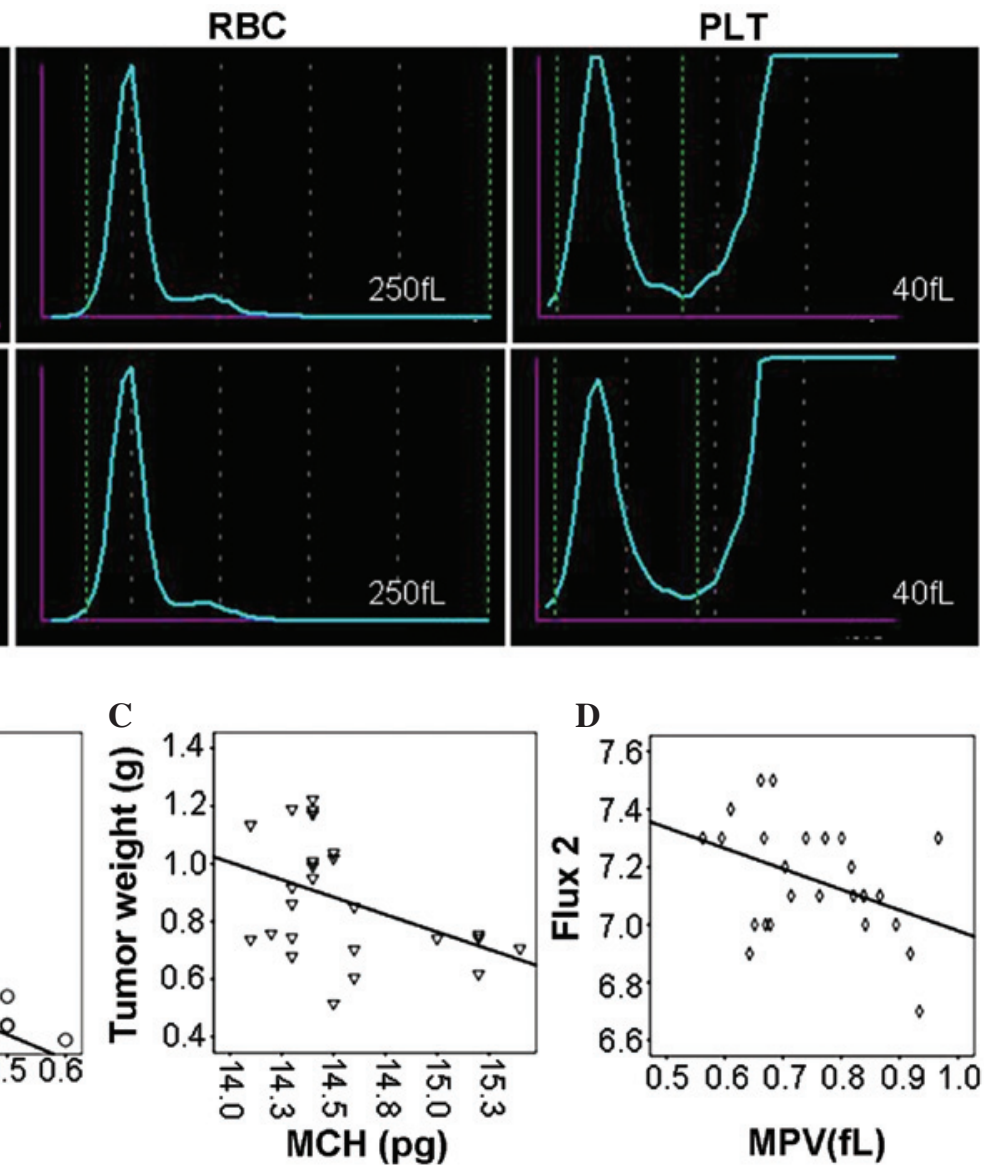

Figure 2. Blood cell parameter changes following tumor cell implantation and their correlation with tumor growth and metastasis. (A) Blood cell parameters were altered following tumor cell implantation. Scattergram of WBC count (left), histogram of RBC count (middle) and histogram of PLT count (right) detected using a Sysmex XT-2000i automated hematology analyzer. Correlations between (B) BASO\% and lung metastases, (C) MCH and tumor weight and (D) MPV and blood perfusion in Flux 2. WBC, white blood cell; RBC, red blood cell; PLT, platelet; BASO\%, ratio of basophils; MCH, mean corpuscular hemoglobin; MPV, mean platelet volume; Flux 2, blood flow in adjacent normal tissue; SSC, side scatter; FSC, forward scatter.

tumors (Flux 2) was markedly increased compared with the value for tumor tissue at week 2 (Fig. 1C).

Distribution of WBCs in tumor-bearing mice. WBC count is the measure of leukocytes cells/ $\mu$ l blood. Blood testing revealed that the average $\mathrm{WBC}$ count of tumor-bearing mice was increased to $\sim 31$ times the normal range (Table II; Fig. 2A). In addition, EO\# increased but EO\% decreased following tumor cell implantation. By contrast, BASO\% and BASO\# were limited in normal mice and increased in tumor-bearing mice. In addition, the lymphocyte number was out of the detection range of Sysmex XT-2000i automated hematology analyzer following tumor cell implantation. Dox treatment did not significantly alter the WBC distribution in tumor-bearing mice. Using Pearson's correlation coefficient analysis to analyze the correlation between WBC parameters, tumor weight and lung metastasis in 25 samples, the results demonstrated an inverse association between lung metastatic number and $\mathrm{BASO} \%$ ( $\mathrm{r}=-0.512 ; \mathrm{P}=0.009)$ (Fig. 2B).

Characterization of red blood cells in tumor-bearing mice. Routine blood test results indicated tumor-associated acute anemia in mice, characterized as decreased RBC, HGB, $\mathrm{HCT}, \mathrm{MCH}$ and RDW-CV values compared with normal 
mice $(\mathrm{P}<0.05)$ (Table III; Fig. 2A). In addition, Dox treatment was demonstrated to significantly increased the levels of $\mathrm{MCH}(\mathrm{P}<0.01)$ and RDW-CV $(\mathrm{P}<0.01)$ compared with the model group. Pearson's correlation coefficient analysis revealed an inverse association between primary tumor weight and $\mathrm{MCH}$ ( $\mathrm{r}=-0.425 ; \mathrm{P}=0.034$ ) (Fig. 2C).

Characterization of platelets in tumor-bearing mice. In 4T1 tumor-bearing mice, values of platelet parameters, including PLT, PCT, PDW, MPV and P-LCR\%, were significantly different from those in normal mice. Platelet parameters were all significantly increased following tumor cell implantation compared with the normal group $(\mathrm{P}<0.05)$. In addition, PLT and PCT were decreased following Dox treatment compared with the model group, although these differences were not significant (Table IV; Fig. 2A). Furthermore, correlation coefficient analysis revealed a negative correlation between Flux 2 at week 3 and MPV (r=-.412; P=.041) (Fig. 2D).

\section{Discussion}

4T1 is an animal model for stage IV human breast cancer, which is able to spontaneously produce highly metastatic tumors that are known to metastasize to the lung, liver, lymph nodes and brain in BALB/c mice (24). The present study aimed to evaluate the change of primary tumor weight, lung metastasis, blood cell parameters and blood perfusion following tumor cell implantation and Dox treatment. The results indicated that the tumor-bearing mice exhibited higher WBC and PLT counts as well as a lower RBC count and $\mathrm{MCH}$. This therefore indicated the presence of tumor-induced thrombosis and acute anemia. In addition, the present study evaluated the described parameters of 30 mice ( 5 normal) and analyzed the associations among the variables. The results demonstrated that the number of visceral lung metastatic sites had a strong negative association with $\mathrm{BASO} \%$; in addition, an inverse association was detected between $\mathrm{MCH}$ and the primary tumor weight.

Existing knowledge and further mechanistic studies have suggested platelets and their functions to be a novel avenue for anti-metastatic therapy $(25,26)$. Clinical studies have indicated that metastatic malignancy or lung malignancy confers a higher risk of cancer-associated venous thromboembolism (VTE) recurrence compared with that of patients with localized malignancy; the occurrence of thromboembolism in a cancer patient has been reported to significantly increase the risk of mortality (27). A review by Louzada et al suggested that VTE recurrence rate according to tumor stage was associated with an increased risk for patients with metastatic malignancy compared with patients with localized disease (relative risk, 1.36; $95 \%$ confidence interval, 1.06-1.74; $\mathrm{P}=0.01$ ) (28). In the present study, a marked increases in platelet-associated parameters, including PLT, PCT, MPV, PDW, PLCR $(\mathrm{P}<0.05)$, and decreases in local blood perfusion were apparent; this therefore suggested that the local blood perfusion of tumor and platelet parameters were associated with primary tumor growth and lung metastasis in a mouse 4T1 breast cancer metastasis model.

Tumor cell-associated platelet aggregation and microthrombus formation occurs when tumor cells become trapped in the microvasculature of a distant organ. Disseminated tumor cells are surrounded by non-activated platelets; once activated, platelets promote metastasis formation by releasing specific growth factors, including platelet-derived growth factor (PDGF) and P-selectin (13). P-selectin is an adhesion molecule expressed by activated platelets and endothelial cells; the increase of circulating PLT leads to the over expression of P-selectin, which subsequently promotes thrombogenic platelet interactions with tumor cells via P-selectin $(29,30)$. In addition, platelets release PDGF, which is a potent inducer of tumor cell invasion (31). Therefore, high platelet counts were reported to be associated with tumor progression and poor prognosis in numerous types of cancer $(32,33)$.

There is increasing evidence for the potential association between acute anemia and tumor growth in clinical practice. The European Cancer Anemia Survey (ECAS) provided data stating that anemia [hemoglobin $(\mathrm{Hb}),<12 \mathrm{~g} / \mathrm{dl}$ ] was detected in $30.4 \%$ of breast cancer patients and $49.1 \%$ of gynecologic cancer patients. Overall, it was reported that at certain points during this survey, $62.4 \%$ of breast cancer patients and $81.4 \%$ of gynecologic cancer patients showed clinical symptoms of anemia (34). In addition, for breast and gynecologic cancer patients, a marked association was detected between low $\mathrm{Hb}$ level and poor performance status (World Health Organization criteria) (35). The data presented by ECAS emphasized the importance of increased awareness of the negative effects of anemia on cancer patients and optimal anemia management in order to ensure the maximum quality of life for patients. $\mathrm{MCH}$ is a marker that indicates the concentration of hemoglobin per RBC (36). The present results demonstrated that when the primary tumor weight was increased, $\mathrm{MCH}$ was decreased.

Leukocytosis, or elevated WBC count, is a commonly encountered laboratory finding. In the present study, WBCs in tumor-bearing mice had a significantly higher baseline mean $\left(313.49 \pm 114.69 \times 10^{9} / 1\right)$ compared with that of normal mice $\left(10.24 \pm 1.57 \times 10^{9} / 1\right)$. Of note, the present study revealed an inverse association between BASO\% and lung metastasis in tumor-bearing mice. Basophils, a type of WBC, which have an important role in allergic disorders, have not been extensively studied; this may be due to their dispersed distribution in hematopoietic organs, as the only account for $<1 \%$ of all blood leukocytes, as well as their limited longevity (37). Basophils are involved in numerous immune reactions, including the initiation of Th2 differentiation, which was reported to be closely associated with cancer metastasis (38). Therefore, understanding the potential action of basophils in the cancer process is critical. A previous study suggested that the assessment of circulating basophils may provide important prognostic information in cancer patients (39). The present study indicated that the presence of basophils predicted tumor growth in mice. In cases where the BASO \% was higher, as in verified metastasis, the number of lung metastatic sites was decreased.

The results of the present study revealed that among breast cancer-bearing mice, widespread cancer-associated thrombosis and acute anemia were observed. In addition, inverse associations between $\mathrm{MHC}$ and tumor weight, as well as BASO \% and lung metastasis were identified. The present study was limited due to a relatively small sample size and only one determination of BASO $\%$ using blood cell analysis. It is therefore recommended that a prospective study should be performed to analyze serial measurements of BASO levels 
and activity as well as the level of tumor metastatic-associated factors during tumor metastasis progress, in order to further determine the association of $\mathrm{BASO} \%$ and lung metastasis in a mouse breast cancer model.

\section{Acknowledgements}

The present study was supported by grants from National Science Foundation of China (nos. 81102852 and 81473575 to Dr Jian-Li Gao, and no. 81202972 to Dr Qi-Yang Shou), the Zhejiang Provincial Key Laboratory Project (no. 2012E10002 to Professor Gui-Yuan Lyu) and the Young Academic Leaders Project of High School in Zhejiang Province (no. pd2013208 to Dr Jian-Li Gao, www.zjedu.gov.cn/news/24455.html).

\section{References}

1. Maruvka YE, Tang M and Michor F: On the validity of using increases in 5-year survival rates to measure success in the fight against cancer. PLoS One 23: e83100, 2014.

2. Herrick AL and Clark S: Quantifying digital vascular disease in patients with primary Raynaud's phenomenon and systemic sclerosis. Ann Rheum Dis 57: 70-78, 1998.

3. Joyce JA and Pollard JW: Microenvironmental regulation of metastasis. Nat Rev Cancer 9: 239-252, 2009.

4. van der Hulle T, den Exter PL, Kooiman J, van der Hoeven JJ, Huisman MV and Klok FA: Meta-analysis of the efficacy and safety of new oral anticoagulants in patients with cancer-associated acute venous thromboembolism. J Thromb Haemost 12: 1116-1120, 2014.

5. Kuderer NM, Ortel TL and Francis CW: Impact of venous thromboembolism and anticoagulation on cancer and cancer survival. J Clin Oncol 27: 4902-4911, 2009.

6. Khorana AA and Connolly GC: Assessing risk of venous thromboembolism in the patient with cancer. J Clin Oncol 27 4839-4847, 2009.

7. Khorana AA and Fine RL: Pancreatic cancer and thromboembolic disease. Lancet Oncol 5: 655-663, 2004

8. Labelle M, Begum S and Hynes RO: Direct signaling between platelets and cancer cells induces an epithelial-mesenchymallike transition and promotes metastasis. Cancer Cell 20: 576-590, 2011.

9. Gao JL, Ji X, He TC, et al: Tetrandrine suppresses cancer angiogenesis and metastasis in 4T1 tumor-bearing mice. Evid Based Complement Alternat Med 2013: 265061, 2013

10. Vadasz B, Chen P, Yougbaré I, et al: Platelets and platelet alloantigens: Lessons from human patients and animal models of fetal and neonatal alloimmune thrombocytopenia. Genes \& Diseases (In Press): 2015.

11. Erpenbeck L and Schon MP: Deadly allies: The fatal interplay between platelets and metastasizing cancer cells. Blood 115 3427-3436, 2010

12. Kim YJ, Borsig L, Varki NM and Varki A: P-selectin deficiency attenuates tumor growth and metastasis. Proc Natl Acad Sci USA 95: 9325-9330, 1998.

13. Gay LJ and Felding-Habermann B: Contribution of platelets to tumour metastasis. Nat Rev Cancer 11: 123-134, 2011.

14. Kahramanca S, Kaya O, Ozgehan G, et al: Are neutrophil-lymphocyte ratio and platelet-lymphocyte ratio as effective as Fournier's gangrene severity index for predicting the number of debridements in Fourner's gangrene? Ulus Travma Acil Cerrahi Derg 20: 107-112, 2014.

15. Min B, Brown MA and Legros G: Understanding the roles of basophils: breaking dawn. Immunology 135: 192-197, 2012.

16. Schmitt E, Meuret G and Stix L: Monocyte recruitment in tuberculosis and sarcoidosis. Br J Haematol 35: 11-17, 1977.

17. Kao SC, Pavlakis N, Harvie R, et al: High blood neutrophil-to-lymphocyte ratio is an indicator of poor prognosis in malignant mesothelioma patients undergoing systemic therapy. Clin Cancer Res 16: 5805-5813, 2010.
18. Use and Care of Laboratory Animals, Zhejiang province: www.sydw.zj.cn/com-apply-singup/com-applyfeeds/2014-10-30-02-17-43/326-2014-10-28-02-41-40.html. Accessed August 17, 2009.

19. Rastegar F, Gao JL, Shenaq D, et al: Lysophosphatidic acid acyltransferase $\beta$ (LPAAT $\beta$ ) promotes the tumor growth of human osteosarcoma. PLoS One 5: e14182, 2010.

20. Gao JL, Lv GY, He BC, et al: Ginseng saponin metabolite 20(S)-protopanaxadiol inhibits tumor growth by targeting multiple cancer signaling pathways. Oncol Rep 30: 292-298, 2013.

21. He BC, Gao JL, Luo X, et al: Ginsenoside Rg3 inhibits colorectal tumor growth through the down-regulation of $\mathrm{Wnt} / \beta$-catenin signaling. Int J Oncol 38: 437-445, 2011.

22. Opazo Saez AM, Mosel F, Nurnberger J, et al: Laser Doppler imager (LDI) scanner and intradermal injection for in vivo pharmacology in human skin microcirculation: Responses to acetylcholine, endothelin-1 and their repeatability. Br J Clin Pharmacol 59: 511-519, 2005.

23. Rack B, Schindlbeck C, Juckstock J, et al: Circulating tumor cells predict survival in early average-to-high risk breast cancer patients. J Natl Cancer Inst 106: 2014.

24. Tao K, Fang M, Alroy J and Sahagian GG: Imagable 4T1 model for the study of late stage breast cancer. BMC cancer 8: 228, 2008.

25. Yu LX, Yan L, Yang W, et al: Platelets promote tumour metastasis via interaction between TLR4 and tumour cell-released highmobility group box1 protein. Nat Comm 5: 5256, 2014.

26. SuMC,ChengCY and Wang PC: Aneural-network-based approach to white blood cell classification. ScientificWorldJournal 2014: 796371, 2014

27. Médioni J, Guastalla JP and Drouet L: Thrombosis and breast cancer: Incidence, risk factors, physiopathology and treatment. Bull Cancer 99: 199-210, 2012 (In French).

28. Louzada ML, Majeed H, Dao V and Wells PS: Risk of recurrent venous thromboembolism according to malignancy characteristics in patients with cancer-associated thrombosis: A systematic review of observational and intervention studies. Blood Coagul Fibrinolysis 22: 86-91, 2011.

29. Mezouar S, Darbousset R, Dignat-George F, Panicot-Dubois L and Dubois C: Inhibition of platelet activation prevents the P-selectin and integrin-dependent accumulation of cancer cell microparticles and reduces tumor growth and metastasis in vivo. Int J Cancer 136: 462-475, 2015.

30. Ludwig RJ, Schön MP and Boehncke WH: P-selectin: A common therapeutic target for cardiovascular disorders, inflammation and tumour metastasis. Expert Opin Ther Targets 11: 1103-1117, 2007

31. Steller EJ, Raats DA, Koster J, et al: PDGFRB promotes liver metastasis formation of mesenchymal-like colorectal tumor cells. Neoplasia 15: 204-217, 2013.

32. Lin MS, Huang JX, Zhu J and Shen HZ: Elevation of platelet count in patients with colorectal cancer predicts tendency to metastases and poor prognosis. Hepatogastroenterology 59: 1687-1690, 2012

33. Sasaki K, Kawai K, Tsuno NH, Sunami E and Kitayama J: Impact of preoperative thrombocytosis on the survival of patients with primary colorectal cancer. World J Surg 36: 192-200, 2012.

34. Barrett-Lee P, Bokemeyer C, Gascón P, et al: Management of cancer-related anemia in patients with breast or gynecologic cancer: New insights based on results from the European Cancer Anemia Survey. Oncologist 10: 743-757, 2005.

35. Yadav D, Mishra M, Joseph AZ, et al: Status of antioxidant and lipid peroxidation in type 2 diabetic human subjects diagnosed with and without metabolic syndrome by using NCEP-ATPIII, IDF and WHO criteria. Obes Res Clin Practice 9: 158-167, 2015.

36. Kemal Y, Demirag G, Bas B, Onem S, Teker F and Yucel I: The value of red blood cell distribution width in endometrial cancer. Clin Chem Lab Med 53: 823-827, 2015.

37. Arinobu Y, Iwasaki $\mathrm{H}$ and Akashi K: Origin of basophils and mast cells. Allergol Int 58: 21-28, 2009.

38. Sokol CL, Barton GM, Farr AG and Medzhitov R: A mechanism for the initiation of allergen-induced T helper type 2 responses. Nat Immunol 9: 310-318, 2008.

39. Valastyan S and Weinberg RA: Tumor metastasis: Molecular insights and evolving paradigms. Cell 147: 275-292, 2011. 